\title{
Automatic prediction of chronic obstructive pulmonary disease exacerbations through home telemonitoring of symptoms ${ }^{1}$
}

\author{
M.A. Fernández-Granero ${ }^{\mathrm{a}, *}$, D. Sánchez-Morillo ${ }^{\mathrm{a}}$, A. León-Jiménez ${ }^{\mathrm{b}}$ and L.F. Crespo ${ }^{\mathrm{a}}$ \\ ${ }^{a}$ Biomedical Engineering and Telemedicine Research Group, University of Cádiz, Cádiz, Spain \\ ${ }^{\mathrm{b}}$ Pulmonology and Allergy Unit, Puerta del Mar University Hospital, Cádiz, Spain
}

\begin{abstract}
Chronic Obstructive Pulmonary Disease (COPD) is a progressive disease of the lung with a great prevalence and a remarkable socio-economic impact on patients and health systems. Early detection of exacerbations could diminish the adverse effects on patients' health and cut down costs burdened on patients with COPD. A group of 16 patients were telemonitored at home using a novel electronic daily symptoms questionnaire during a 6-months field trial. Recorded data were used to train and validate a Probabilistic Neural Network (PNN) classifier in order to enable the automatic prediction of exacerbations. The proposed system was able to predict COPD exacerbations early with a margin of $4.8 \pm 1.8$ days (average $\pm \mathrm{SD}$ ). Detection accuracy was $80.5 \%$ (33 out of 41 exacerbations were early detected); $78.8 \%$ (26 out of 33) of theses detected events were reported exacerbation and $87.5 \%$ (7 out of 8 ) were unreported episodes. The proposed questionnaire and the designed automatic classifier could support the early detection of COPD exacerbations of benefit to both physicians and patients.
\end{abstract}

Keywords: Chronic Obstructive Pulmonary Disease, exacerbation, telehealth, symptoms, questionnaire, early detection, Probabilistic Neural Network, telemonitoring

\section{Introduction}

Chronic Obstructive Pulmonary Disease (COPD) is a progressive disease of the lung with a great prevalence and a remarkable socio-economic impact on patients and health systems [1,2]. Although findings from studies done over the past 20 years have suggested a downward trend in COPD mortality [3], COPD is still a leading cause of death in the world. Prevalence of COPD varies from country to country [4]. In Spain, the prevalence in 40 to 80 -year-old people has been estimated about $10.2 \%$ [5].

\footnotetext{
${ }^{1}$ The work presented here was carried out in collaboration between all authors: they all defined the research theme, read abstracts, selected reviews for full text assessment, reviewed related papers, designed, conducted the study, interpreted its results, revised the manuscript and approved the final version. MAFG and DSM managed the review process. MAFG and DSM drafted the full article and revised it critically for intellectual content. MAFG and DSM carried out the computerized processing of data and ALJ gave expert respiratory input and ALJ and LFC contributed to study conceptualization and refining on outcome measures.

${ }^{*}$ Corresponding author: M.A. Fernández-Granero, Biomedical Engineering and Telemedicine Research Group, University of Cádiz, Cádiz, Spain. Tel.: +0034956015155; E-mail: ma.fernandez@uca.es.
} 
COPD poses a significant economic burden on society. The largest proportion of the disease-related cost is attributable to a small percentage of patients (those severe cases) because most expenditure is associated with hospitalizations by Acute Exacerbations of COPD (AECOPD) [6]. Global Initiative for Chronic Obstructive Lung Disease (GOLD) guidelines define a COPD exacerbation as "an event in the natural course of the disease characterized by a change in the patient's baseline dyspnea, cough, and/or sputum that is beyond normal day-to-day variations, is acute in onset, and may warrant a change in medication in a patient with underlying COPD" [7]. Normally, AECOPD occurs in a batchwise manner about twice or three times per year, depending on COPD severity grade and anamnesis, and goes along with a worsening state of the patient health, which exerts adverse effects on the patient's physical efficiency, quality of life and independence. Not rarely, AECOPD also leads to hospitalization of the patient. In these cases, socio-economical cost arises. Consequently, early detection of exacerbations could diminish the adverse effects on patients' health and cut down costs burdened on patients with COPD by enabling an early treatment and therefore a reduced recovery time and risk of hospitalization [8].

To cope with the growing shortage in care capabilities, a personalized out-of-hospital healthcare model which supports independent lifestyle, chronic disease management, abnormality detection ability, timely and accurate healthcare services and emergency handling capability is highly desirable [9]. The telemonitoring of symptoms on a daily basis can be a sustainable alternative for the close followup of patients with COPD. Prediction of AECOPD has been recently studied and reported [10-20]. According to recent systematic reviews, emergency admissions due to AECOPD could be reduced through home telemonitoring [21]. Furthermore, telehealth interventions have proven to reduce mortality [22]. However, more evidence on the benefits of telehealth strategies is required [23].

In this study, a multimodal mobile health system, specially designed by and for patients with COPD was used to determine the feasibility of the prediction of AECOPD on a day-to-day basis [24]. A novel questionnaire for the early detection of AECOPD was evaluated during a 6-months field trial. Recorded data were processed using pattern recognition methods and accuracy in predicting AECOPD was assessed.

\section{Patients and methods}

\subsection{Patients}

The study samples consisted of 16 COPD patients recruited in the University Hospital Puerta del Mar of Cadiz (Spain) from the Pulmonology and Allergy Unit. Patients signed an informed consent after the study was approved by the Hospital's research ethics committee. Inclusion criteria included participants with at least one AECOPD with associated hospital admission or two AECOPD treated with oral antibiotics or corticosteroids in the previous year. Patients were all aged over 60 years and had a diagnosis of COPD with severity II, III or IV confirmed by spirometry (GOLD guidelines).

\subsection{Telemonitoring system, reporting of symptoms y automatic detection of exacerbations}

The system emulated a medical consultation including a medical interview (Figure 1). A telemedical server with an electronic patient record was located at the Hospital. Patients were equipped with a Dedicated mobile Device $(D m D)$ with speech and touch interfaces to daily respond to a questionnaire. The remote server was connected to each $D m D$ through a secure private IP-based wireless telemedi- 


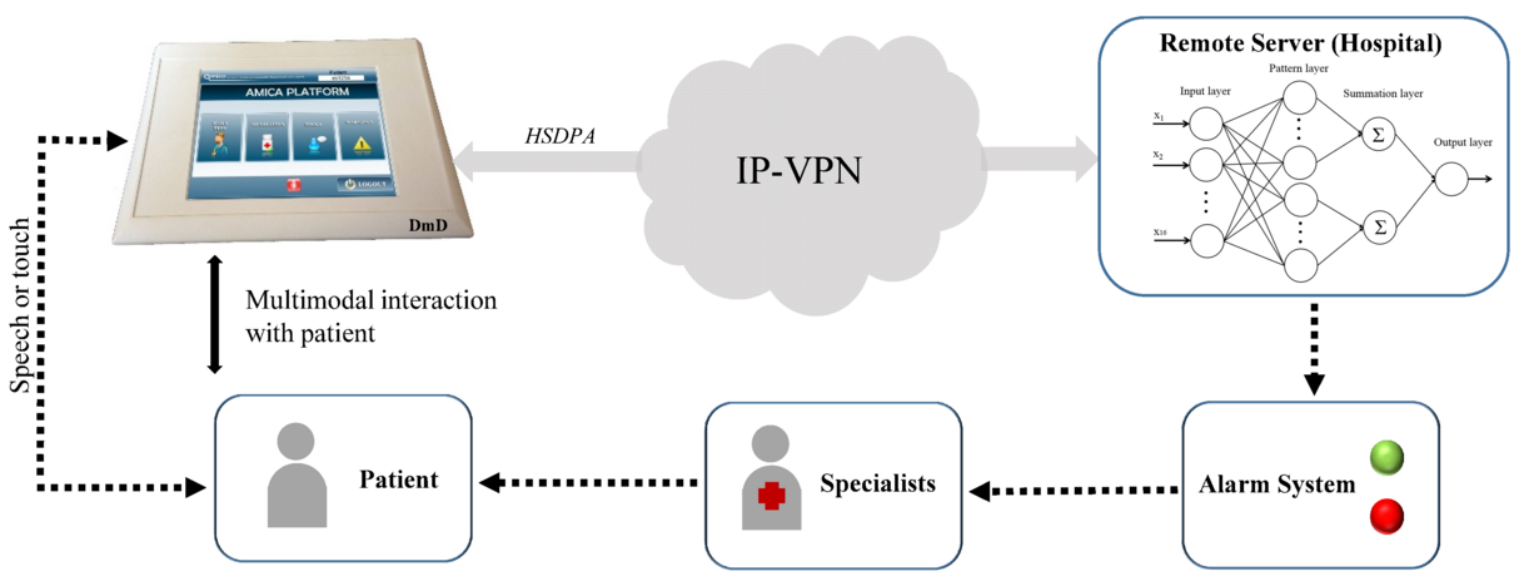

Fig. 1. Home telemonitoring system for patients with COPD.

cine network [25]. A multimodal interface guided the patients through recording their symptoms on a daily basis according to the implemented Automated Questionnaire for the early detection of AECOPD (AQCE) [24]. Exacerbations were defined according to Anthonisen criteria [26].

A probabilistic neural network (PNN) was used for classification [27]. A PNN is an implementation of the kernel discriminant analysis algorithm in which the operations are organized into a multilayered feed-forward network.

The network architecture presents four fully interconnected layers. The input layer has as many neurons as the number of features in the training set. The pattern layer has one neuron for each case in the training data set. Each neuron estimates the Probability Density Function (PDF) of the input vector using the Parzen window method [28]. The Gaussian function was used as Parzen window. The summation layer has two neurons in the case of two classes. Each of these two nodes estimates a class conditional PDF using a mixture of Gaussian kernels. The output layer bases decision on the Bayes optimal decision rule.

The training process of a PNN is essentially the act of determining the value of the smoothing parameter $(\sigma)$. It controls the size and shape of the Gaussian PDF. A separate sigma value for each predictor variable was used in the model. The optimal $\sigma$ values were estimated with the conjugate gradient algorithm. During training, the residual error of the model using the weight values was estimated by the iteration. If the error did not improve after 1000 consecutive iterations, the conjugate gradient process would be stopped. A maximum of 5000 total iterations were defined. Jackknife method was used to grade the performance for each $\sigma$ value during the optimization process. If the residual error of the model was less than $10^{-8}$, convergence would be assumed and the conjugate process would be stopped.

Twelve predictor variables processed from AQCE and four additional parameters (three-day moving average applied to the total score and average scores for symptoms associated with major, minor and complementary symptoms) were used as inputs for the classifier. An alarm state was programmed to be triggered after two consecutive days with a positive output in the PNN-classifier.

Ten-cross validation was used to build outcome prediction model. Missing data were processed using backward and forward imputation [29]. Receiver Operating Characteristic (ROC) analysis was carried out [30]. Performance was assessed according to sensitivity, specificity, accuracy, confusion matrix, positive predictive value and negative predictive value. MathWorks MATLAB ${ }^{\circledR}$ was used for statistical analysis. 


\section{Results}

Table 1 shows the clinical and demographic characteristics of the participants.

As shown in Figure 2, 15 patients were included in the final study and one patient was excluded. During the trial, the patients suffered from 41 episodes that matched Anthonisen criteria and therefore were considered as AECOPD periods. Furthermore, 52-time periods without meeting Anthonisen criteria were counted and therefore defined as non-AECOPD periods.

Table 1

Demographic characteristics of the study group including clinical data summarized after the field trial

\begin{tabular}{ll}
\hline Variable & $\mathrm{N}(\%)$ \\
\hline Age (years) & $70.2 \pm 6.6$ \\
Male (\%) & $93 \%(14)$ \\
COPD Stage (\%) & \\
Stage I ( $>80 \%)$ & $0 \%(0)$ \\
Stage II $(50 \%-79 \%)$ & $13.3 \%(2)$ \\
Stage III (30-49\%) & $46.7 \%(7)$ \\
Stage IV $(<30 \%)$ & $40.0 \%(6)$ \\
Exacerbations per patient $(\%)$ & \\
None & $13.3 \%(2)$ \\
1 & $20 \%(3)$ \\
2 or 3 & $20 \%(3)$ \\
4 or more & $46.6 \%(7)$ \\
Hospitalizations $(\%)$ & \\
0 & $66.6 \%(10)$ \\
1 & $20 \%(3)$ \\
2 or more & $13,3 \%(2)$ \\
\hline
\end{tabular}

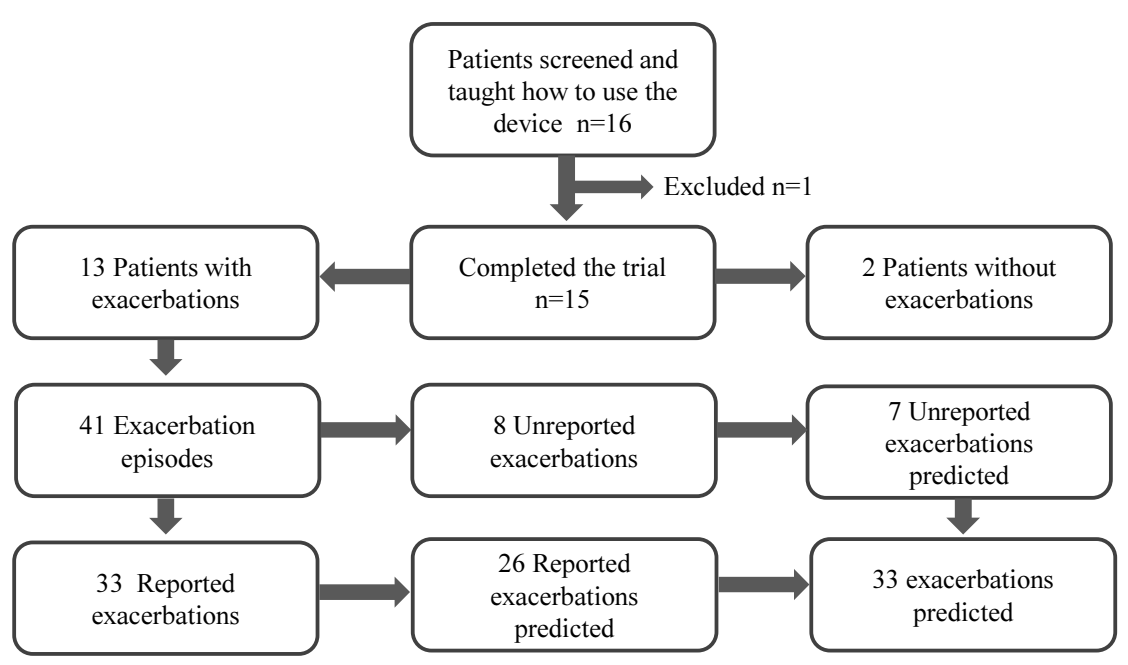

Fig. 2. Flowchart with detailed information on patient participation, drop out and exacerbations throughout the study period. 


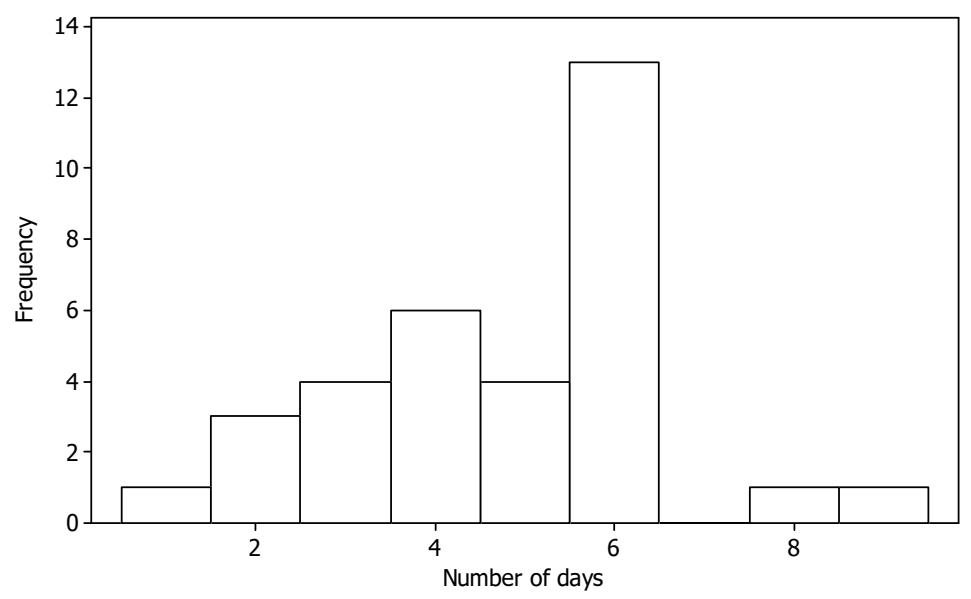

Fig. 3. Histogram of prediction margins based on performance of the PNN classifier.

Table 2

Classifiers Performance Evaluation

\begin{tabular}{ll}
\hline Time periods & $94(41+53)$ \\
True Positives (TP) & $33(35 \%)$ \\
False Positives (FP) & $3(3 \%)$ \\
True Negatives (TN) & $50(53 \%)$ \\
False Negatives (FN) & $8(8.5 \%)$ \\
Accuracy & $88.3 \%$ \\
Sensitivity & $80.5 \%$ \\
Specificity & $94.34 \%$ \\
Positive Predictive Value (PPV) & $91.67 \%$ \\
Negative Predictive Value (NPV) & $86.21 \%$ \\
\hline
\end{tabular}

In this experiment, 33 out of the 41 episodes were event-based exacerbations, i.e. exacerbations for which antibiotic or corticosteroid treatment was initiated. The rest 8 out of 41 AECOPD were symptom-based exacerbations, i.e. episodes which were not reported to physicians but were detected through symptom monitoring. 2104 questionnaires were received during the trial. Each sample was represented by sixteen input features and one output for training and validating the PNN classifier. Operating point in the ROC curve was selected to minimize the number of false positives results.

Figure 3 shows the histogram of predictions for the detection of AECOPD. Table 2 details performance parameters of the resulting classifier. It was observed that $80.5 \%$ (33 out of 41) AECOPD were early detected, $78.8 \%$ (26 out of 33 ) were reported AECOPD and $87.5 \%$ (7 out of 8 ) were unreported AECOPD. The alarm system had only 3 false positives. The system was able to early detect AECOPD with $4.8 \pm 1.8$ average \pm SD days prior to the day when the patients met Anthonisen criteria.

\section{Discussion}

Efficiency of the proposed automated classifier for the early detection of AECOPD was assessed in a 6-months trial with 15 patients. A PNN classifier was trained and validated using the responses to an 
electronic questionnaire designed for the daily remote monitoring of patients with COPD. As a result, 33 out of 41 AECOPD were predicted early within 4.8 days prior to onset as average.

Systems based on patient symptom diary cards usually do not provide accurate information on the severity and duration of the episodes [31]. Recently, the EXAcerbation of Chronic Pulmonary Disease Tool (EXACT) [32] has been developed and evaluated. Nevertheless, although EXACT can determine the frequency, severity and duration of AECOPD, a poor performance in AECOPD detection has been reported. In this regards, the strategy described in this manuscript increases the precision in the detection of AECOPD and enables the estimation in the duration and severity of episodes.

Furthermore, it allows detecting unreported AECOPD. And 7 out of 8 unreported AECOPD were detected during the field trial. Detection of unreported AECOPD allows the patients to initiate a selfmanagement plan or to request medical attention. To sum up, self-managing of the disease is empowered and clinical efficiency is improved.

Definition of AECOPD is controversial [32], mainly due to the heterogeneous nature of exacerbations. In our study, an exacerbation was established according to Anthonisen criteria (symptom-based exacerbation). However, the results are consistent with our previous study where an AECOPD was defined as a non-programmed visit because of respiratory symptoms to the emergency unit, to the primary care center or to the specialist (event-based exacerbation) [33]. In that study, a Radial Basis Function classifier (RBF) was able to detect AECOPD within 5.3 days, as average, prior to the day when the patients required medical attention. Consequently, the proposed system is highly robust to AECOPD definition, whether based on symptoms or events.

With the encouragement of the initial results, this experience has to be expanded to include a larger number of patients to generalize results. Furthermore, a greater number of participants would avoid a possible optimistic bias by using Leave-One-Subject-Out Cross Validation (LOSO-CV).

Finally, some studies have raised questions about the usefulness of telemonitoring systems for COPD patients and about their effectiveness [22]. It will remain a priority for the future to develop methods to relate symptoms to objective elements that will provide clinicians with the confidence needed to make a specific diagnosis. These objective elements could be extracted from physiological signals. But problems related to the actual "ease-of-use" of devices need to be overcome [31]. Ease of use is directly related to compliance with daily tasks such as reporting of symptoms. In this regard, our work differs from other proposals in that it is, to our knowledge, the first questionnaire specifically designed for patients with COPD, to be supported on a computing device that provides an interface for multimodal patient interaction [24]. This not only improves patient compliance, but accessibility for patients poorly adapted to new technologies.

\section{Conclusion}

This study aimed at an early detection of COPD exacerbations through home telemonitoring of symptoms. The proposed system was able to early predict COPD exacerbations with a margin, as average, of 4.8 days. Detection accuracy of $80.5 \%$ and a low rate of false positive results were achieved. The designed questionnaire, the trained and validated automatic classifier could support the early detection of COPD exacerbations of benefit to both physicians and patients. 


\section{Acknowledgment}

This work was supported in part by the Ambient Assisted Living (AAL) E.U. Joint Programme, by grants from Ministerio de Educación y Ciencia of Spain and Instituto de Salud Carlos III under Projects PI08/90946 and PI08/90947.

\section{References}

[1] C. Jinjuvadia, S. Liangpunsakul, R. Jinjuvadia, A. Soubani and R. Supakul, Trends in outcomes, hospitalization costs, financial burden, and mortality for Chronic Obstructive Pulmonary Disease (COPD) in the United States from 20022010, Am. J. Respir. Crit. Care Med. 189 (2014), A3721.

[2] A.A. Dalal, S. Nagar, A.O. D'Souza, M. Shah, E. Farrelly and J. Patel, Economic burden of frequently and infrequently exacerbating patients with a diagnosis of COPD, Am. J. Respir. Crit. Care Med. 187 (2013), A1437.

[3] J.L. López-Campos, M. Ruiz-Ramos and J.B. Soriano, Mortality trends in chronic obstructive pulmonary disease in Europe, 1994-2010: a joinpoint regression analysis, The Lancet Respiratory Medicine 2 (2014), 54-62.

[4] A.S. Buist, M.A. McBurnie, W.M. Vollmer et al., International variation in the prevalence of COPD (The BOLD Study): a population-based prevalence study, Lancet 370 (2007), 741-749.

[5] M. Miravitlles, J.B. Soriano, F. García-Río, L. Muñoz, E. Duran-Tauleria, G. Sanchez, V. Sobradillo and J. Ancochea, Prevalence of COPD in Spain: impact of undiagnosed COPD on quality of life and daily life activities, Thorax 64 (2009), 863-868.

[6] P.N. Perera, E.P. Armstrong, D.L. Sherrill and G.H. Skrepnek, Acute exacerbations of COPD in the United States: inpatient burden and predictors of costs and mortality, COPD, Journal of Chronic Obstructive Pulmonary Disease 9 (2012), $131-141$

[7] J. Vestbo et al., Global strategy for the diagnosis, management, and prevention of chronic obstructive pulmonary disease: GOLD executive summary, Am. J. Respir. Crit. Care Med. 187 (2014), 347-365.

[8] T.M. Wilkinson, G.C. Donaldson, J.R. Hurst, T.A. Seemungal and J.A. Wedzicha, Early therapy improves outcomes of exacerbations of chronic obstructive pulmonary disease, Am. J. Respir. Crit. Care Med. 169 (2004), 1298-1303.

[9] J. Li, H. Zhou, D. Zuo, K.M. Hou and C. De Vaulx, Ubiquitous health monitoring and real-time cardiac arrhythmias detection: a case study, Bio-Medical Materials and Engineering 24 (2014), 1027-1033.

[10] A.V. Bennett, D. Amtmann, P. Diehr and D.L. Patrick, Comparison of 7-day recall and daily diary reports of COPD symptoms and impacts, Value Health 15 (2012), 466-474.

[11] J.M. Marin, S.J. Carrizo, C. Casanova, P. Martinez-Camblor, J.B. Soriano, A.G. Agusti and B.R. Celli., Prediction of risk of COPD exacerbations by the BODE index, Respir. Med. 103 (2009), 373-378.

[12] G. Segrelles Calvo, C. Gómez-Suárez, J.B. Soriano, E. Zamora, A. González-Gamarra, M. González-Béjar and J. Ancochea, A home telehealth program for patients with severe COPD: The PROMETE study, Respiratory Medicine 108 (2013), 453-462.

[13] D. Singh, J. Kampschulte, J.A. Wedzicha, P.W. Jones, G. Cohuet, M. Corradi, T. Higenbottam, S. Petruzzelli and J. Vestbo, A trial of beclomethasone/formoterol in COPD using EXACT-PRO to measure exacerbations, Eur. Respir. J. 41 (2013), 12-17.

[14] H. Pinnock, J. Hanley, L. McCloughan et al., Effectiveness of telemonitoring integrated into existing clinical services on hospital admission for exacerbation of chronic obstructive pulmonary disease: researcher blind, multicentre, randomised controlled trial, British Medical Journal 347 (2013), f6070.

[15] Z.M. Sund, T. Powell, R. Greenwood and N.A. Jarad, Remote daily real-time monitoring in patients with COPD-A feasibility study using a novel device, Resp. Med. 103 (2009), 1320-1328.

[16] J. Trappenburg, I. Touwen, G. Oene, J. Bourbeau, E. Monninkhof, T. Verheij, J. Lammers and A. Schrijvers, Detecting exacerbations using the Clinical COPD Questionnaire, Health Qual. Life Outcomes 8 (2010), 102.

[17] J. Ure, H. Pinnock, J. Hanley, G. Kidd, E. McCall Smith, A. Tarling, C. Pagliari, A. Sheikh, W. MacNee and B. McKinstry, Piloting tele-monitoring in COPD: a mixed methods exploration of issues in design and implementation, Prim. Care Resp. J. 21 (2012), 57-64.

[18] M. Van der Heijden, P.J. Lucas, B. Lijnse, Y.F. Heijdra and T.R. Schermer, An autonomous mobile system for the management of COPD, J. Biomed. Inform. 46 (2013), 458-469.

[19] E. Walters, J. Walters, K. Wills, A. Robinson and R. Wood-Baker, Clinical diaries in COPD: compliance and utility in predicting acute exacerbations, International Journal of Chronic Obstructive Pulmonary Disease 7 (2012), 427-435. 
[20] J.L. Pecina, G.J. Hanson, H. Van Houten and P.Y. Takahashi, Impact of telemonitoring on older adults health-related quality of life: the Tele-ERA study, Quality of Life Research 22 (2013), 2315-2321.

[21] J. Cruz, D. Brooks and A. Marques, Home telemonitoring effectiveness in COPD: a systematic review, International Journal of Clinical Practice 68 (2014), 369-378.

[22] A. Steventon, M. Bardsley, J. Billings, J. Dixon, H. Doll, S. Hirani et al., Effect of telehealth on use of secondary care and mortality: findings from the Whole System Demonstrator cluster randomised trial, British Medical Journal 344 (2012), e3874.

[23] C.E. Bolton, C.S. Waters, S. Peirce and G. Elwyn, Insufficient evidence of benefit: a systematic review of home telemonitoring for COPD, Journal of evaluation in clinical practice 17 (2011), 1216-1222.

[24] D. Sánchez-Morillo, M. Crespo, A. León and L.F. Crespo Foix, A novel multimodal tool for telemonitoring patients with COPD, Informatics for Health and Social Care 0 (2013), 1-22.

[25] L.C. Foix, D.S. Morillo, M. Crespo, N. Gross, C. Kunze, K. Giokas and J.A. Jimenez, AMICA telemedicine platform: a design for management of elderly people with COPD, IEEE Transactions of Information Technology and Applications in Biomedicine (ITAB), 2009, 1-4.

[26] N.R. Anthonisen, J. Manfreda, C.P. Warren, E.S. Hershfield, G.K. Harding and N.A. Nelson, Antibiotic therapy in exacerbations of chronic obstructive pulmonary disease, Ann. Intern. Med. 106 (1987), 196-204.

[27] D.F. Specht, Probabilistic neural networks, Neural Networks 3 (1990), 109-118.

[28] E. Parzen, On estimation of a probability density-function and mode, Ann. of Math Statistics 33 (1962), 1065-1076.

[29] J.C.A. Trappenburg, E.M. Monninkhof, J. Bourbeau, T. Troosters, A.J.P. Schrijvers, T.H.J. Verheij and Lammers, Effect of an action plan on recovery and health status impact of exacerbations in COPD patients; a multicenter randomized controlled trial, Thorax 66 (2011), 977-984.

[30] C.E. Metz, Basic principles of ROC analysis, Semin. Nucl. Med. 8 (1978), 283-298.

[31] B.R. Celli, J. Vestbo, The EXACT-Pro: Measuring exacerbations of COPD, Am. J. Respir. Crit. Care Med. 183 (2011), 287-291.

[32] A.J. Mackay, G.C. Donaldson, A.R. Patel, R. Singh, B. Kowlessar and J.A. Wedzicha, Detection and severity grading of COPD exacerbations using the exacerbations of chronic pulmonary disease tool (EXACT), Eur. Respir. J. 43 (2014), $735-744$.

[33] M.F. Granero, D.S. Morillo, A. León, M.L. Gordo and L.F. Crespo, Radial-basis-function based prediction of COPD exacerbations, XIII Mediterranean Conference on Medical and Biological Engineering and Computing, Springer International Publishing 41 (2014), 1455-1458. 